\title{
Technologie-Roadmap Prozess-Sensoren 2015+
}

\section{Neue Fassung grundlegend aktualisiert und überarbeitet}

Die Wettbewerbsfähigkeit der Chemisch-Pharmazeutischen Industrie basiert auf der Sicherung der geforderten Produktqualität bei einer optimalen Nutzung von Anlagen, Rohstoffen und Energie. Eine gute Prozessführung unter Einsatz zuverlässiger Prozessautomation sichert hier den globalen Wettbewerbsvorteil. Initiiert von NAMUR und GMA und unter Mitwirkung der Unternehmen ABB, BASF, BTS, BIS Prozesstechnik, E+H, Siemens und der BAM entstand jetzt die grundlegend aktualisierte und überarbeitete TechnologieRoadmap „Prozess-Sensoren 2015+“, die im November 2009 veröffentlicht wurde.

SCHLAGWÖRTER Roadmap / Prozess-Sensoren / Prozessanalytik

\section{The Updated Technology Roadmap Process Sensors 2015+}

The competitiveness of the chemical pharmaceutical industry is based on the protection of the demanded product quality with an optimum use of equipment, raw materials, and energy. A good process guidance at the basis of reliable process automation protects the global competitive advantage. Initiated by NAMUR and GMA and in collaboration with ABB, BASF, BTS, BIS process technology, E+H, Siemens and BAM an updated and revised technology roadmap „Process Sensors 2015+“ was recently created and published in November 2009.

KEYWORDS roadmap / process sensors / process analytics 
MICHAEL MAIWALD, BAM Bundesanstalt für Materialforschung und -prüfung

E in Jubiläum im Jahr 2009 liefert den passenden Rahmen für eine Einleitung: Der Star-Trek-Tricorder wird 40 Jahre alt! Das Gerät aus der Serie Raumschiff Enterprise bzw. Star Trek erlaubt chemische, materialtechnische und medizinische Analysen, um dem Anwender in Verbindung mit einem Zentralcomputer umgehend die resultierenden Diagnosen und Handlungsempfehlungen zu liefern. Was 1969 pure ScienceFiction war, ist heute zum erheblichen Teil greifbare technologische Realität geworden.

„Kommunikation“ war das Motto der NAMUR-Hauptsitzung 2009 in Bad Neuenahr. Die heutige Kommunikationstechnik übertrifft die Zukunftserwartungen von 1969 am genannten Beispiel hinsichtlich Handhabbarkeit, Kompaktheit und interaktive Benutzerführung bereits bei weitem. Die analytische Sensorik und Ferndiagnostik hat in diesen 40 Jahren bedeutende Fortschritte gemacht. Wie lässt sich die Weiterentwicklung von Prozess-Sensorik also weiter antreiben?

\section{BEDARF AN NEUEN PROZESS-SENSOREN}

Eine stets optimale Nutzung von Anlagen, Rohstoffen und Energie in Verbindung mit einer guten Prozessführung sind die wichtigsten Voraussetzungen für globale Wettbewerbsvorteile. Eine zuverlässige Prozessautomation erhöht hier die Prozess- und Anlagensicherheit. Überlegene Verfahren bedingen aber nicht nur die Kontrolle der geforderten Produktqualität post mortem sondern auch ihre präventive Sicherstellung. Damit ist eine ständige Verbesserung des Wissens über den Prozess gefordert.

Neben einem übergreifenden Informationsmanagement beginnt ein solches wissensbasiertes Prozessverständnis für die Verfahrensentwicklung und Produktion bei den ProzessSensoren, die den Prozess transparent machen. Sie sind die „Sinnesorgane der Prozessleittechnik“ für automatisierte Verfahren. Die Spanne zur optimalen Steuerung anspruchsvoller Produktionsprozesse reicht von den klassischen Sensoren, wie z.B. für Temperatur oder Druck, über weitere Messgrößen der Prozessanalysenmesstechnik bis hin zu instrumentell analytischen Techniken der Prozessanalytik. Besonders in der chemischen und pharmazeutischen Reaktionstechnik liefern letztere Instrumente wertvolle spezifische Informationen über Konzentrationen und Eigenschaften interessanter Komponenten des betrachteten Reaktionsoder Trennschritts und deren zeitlichen Verlauf.

\subsection{Dreifacher Nutzen und gedritteltes Risiko}

Mit der Hilfe von anforderungsgerechten Prozess-Sensoren kann aus Sicht der Anwender ein erheblicher Wettbewerbsvorteil erzielt werden, der auch den Geräteherstellern zugutekommt. Die konsequente Weiterentwicklung und ständige Verbesserung der Prozess-Sensorik ist daher eine wichtige Aufgabe von Forschung und Geräteherstellern zusammen mit den Anwendern - im sogenannten „Trialog“. Um die Aufmerksamkeit und Kraft auf die wichtigsten Entwicklungsfelder zu lenken und die Potenziale möglichst schnell zu heben, ist aber eine Fokussierung auf Basis einer realistischen Grundlage notwendig. Durch die Identifizierung und zeitliche Einordnung von technologischen Weiterentwicklungen kann auf allen Seiten das zukünftige Potenzial von Anlagen besser abgeschätzt und eine Investitionsplanung unterstützt werden. Eine Technologie-Roadmap ermöglicht aber auch den Herstellern von Prozess-Sensoren einen Überblick über heutige und zukünftige Anwenderanforderungen. Dieses Wissen versetzt Unternehmen der Automatisierungstechnik in die Lage, eine zielgerichtete Technologieund Produktentwicklung vorzunehmen und daraus Forschungs- und Entwicklungsstrategien abzuleiten. Nun ist es nötig, in gemeinsamen Anstrengungen von Herstellern, Anlagenbetreibern und Forschung (Institute, Hochschulen) die naheliegenden Ziele zu realisieren.

\section{2. „PROZESS-SENSOREN 2015+“; NOCH MEHR ANWENDUNGSBEZUG}

Die 2005 erstmals veröffentlichte Technologie-Roadmap wurde in einem gemeinsamen Projekt der Organisatio- 
nen NAMUR und VDI/VDE- Gesellschaft Mess- und Automatisierungstechnik unter Mitwirkung führender Hersteller und Anwender von Prozess-Sensorik sowie dem Fraunhofer-Institut für Produktionstechnik und Automatisierung (IPA) 2004 erarbeitet. [1, 2] Durch das Zusammenbringen von Technologie- und Marktsicht sowohl aus Anwender- als auch Herstellersicht haben die Verbände den künftigen Handlungsbedarf bzw. das Potenzial im Bereich der Prozess-Sensorik aufgezeigt.

Die chemisch-pharmazeutische Industrie ist für den Standort Deutschland von hoher Bedeutung: Mit ca. 420.000 Beschäftigen erwirtschaftet die Branche einen Umsatz von mehr als 170 Mrd. Euro jährlich (2007). Die Stärkung der Wettbewerbsfähigkeit dieser Branche und ihrer Prozesstechnikhersteller ist damit bereits eine lohnenswerte Aufgabe. Daneben setzen auch weitere wichtige Branchen wie Kraftwerke und Energie, Petrochemie, Papier/Zellstoff, Wasser/Abwasser sowie Nahrungs- und Genussmittel Prozess-Sensoren ein.

Auf der Grundlage der ersten Roadmap haben sich nach Aussagen verschiedener Sensorik-Hersteller bereits eine Reihe konkreter Entwicklungen und Anwendungen ergeben. [3]

\subsection{Gemeinsamer Aufbruch zur Version 1.5}

So wurde auf der NAMUR-Hauptsitzung 2007 angeregt, die bestehende Technologie Roadmap zu aktualisieren und die Betreibersicht noch stärker einzubringen [4]. Der NAMUR-Vorstand erteilte 2008 das Mandat zu einer entsprechenden Überarbeitung und gab damit den Startschuss für diese Überarbeitung und Fortschreibung.

Unter Mitwirkung der Unternehmen ABB, BASF, Bayer Technology Services, BIS Prozesstechnik, Endress+Hauser und Siemens, Vertretern der NAMUR und GMA und unter der Projektleitung der BAM Bundesanstalt für Materialforschung und -prüfung entstand in nur einem Jahr die grundlegend aktualisierte und überarbeitete TechnologieRoadmap „Prozess-Sensoren 2015+“, die nun vorliegt und frei im Internet verfügbar ist [5].

Es war zunächst offen, welchen Umfang eine Überarbeitung haben würde. Aus heutiger Sicht charakterisiert die Bezeichnung „Version 1.5“ die Überarbeitung am besten. Dieses bedeutet, dass im wesentlichen alle wichtigen Kernaussagen der ersten Roadmap validiert werden konnten. Gleichzeitig sind einige neue, nicht weniger wichtige Anforderungen hinzugekommen, die das aktualisierte Papier heute ergänzen und erweitern.

\subsection{Validierung aus der Betreiberperspektive}

Um die Ergebnisse auf eine möglichst anwendungsbezogene Basis zu stellen, wurden über das Projektteam zu Beginn der Überarbeitung mit Hilfe einer gemeinsam konzipierten Umfrage zunächst Informationen von mehr als 100 chemischen Produktionsbetrieben europaweit eingeholt. Aus dem Know-how von erfahrenen Betriebsingenieuren und Herstellervertretern ergab sich darüber eine sehr konkrete und anwenderbezogene Technologie- und Marktsicht auf Entwicklungsziele für neue Sensorik in verfahrenstechnischen Prozessen.

\section{WAS GIBT ES NEUES?}

Sensoren werden für höchst unterschiedlichste Zwecke in der Prozessautomatisierung eingesetzt. Daneben herrscht im chemischen und chemisch-pharmazeutischen Umfeld eine große Heterogenität bezüglich der Anlagen und Verfahren, die in der Regel sehr spezielle Sensorik erfordern. Es ist also eine schwierige Aufgabe, aus dieser Vielfalt ein zusammenfassendes Abbild des Bedarfs für Neues herauszuarbeiten, ohne die Detailsicht zu verlieren und ohne zu grobmaschig für eine technologische Beschreibung zu werden.

\subsection{Im Detail auf das Wesentliche blicken}

Im vorliegenden Projekt wurden durch die Beteiligten erneut Priorisierungen vorgenommen und aus den Gebieten der verfahrenstechnischen Industrie zunächst die Chemische und Pharmazeutische Industrie betrachtet. Aus den möglichen Teilanlagen einer verfahrenstechnischen Anlage wurden schließlich die wichtigsten Verfahrensschritte und Subprozesse als Betrachtungsgegenstand mit höchster Bedeutung für die Entwicklung von ProzessSensoren ausgewählt, wie z.B. Reaktion, Polymerisation, Fermentation, Fällung, Kristallisation, Rektifikation, Filterung, Trocknung und Formulierung. Die Schwerpunkte konnten aus der Umfrage heraus validiert werden.

Für diese Verfahrensschritte konnten die Anforderungen aus Anwendersicht noch detailliert genug beschrieben und in Anforderungsbögen zusammengefasst werden. Dort sind die Applikationen charakterisiert und in ihren typischen Einsatzbedingungen dargestellt. Neben dem einsetzbaren Temperatur- und Druckbereich werden die erforderlichen Messgrößen, Messunsicherheiten und zeitliche Auflösung genannt. Zusätzlich zur Darstellung der Ist-Ausprägung wurden auch Visionen für zukünftige Messaufgaben abgefragt. Die Anforderungsblätter schließen mit möglichen technischen Lösungswegen zur zukünftigen Umsetzung der Messaufgabe inklusive einer Einschätzung der zeitlichen Realisierbarkeit aus Sicht der Hersteller.

\subsection{Thesen und Trends}

Die wichtigsten Ergebnisse der Anwenderabfrage wurden in Kernthesen zusammengefasst. Ein Großteil der Thesen aus der ursprünglichen Roadmap hat auch heute weiter Bestand.

Neben der Forderung nach einer höheren Robustheit und Langzeitstabilität bei niedrigem Instandhaltungsbedarf sowie höherer Genauigkeit wird etwa auch technologische Weitsichtigkeit für neue Prozess-Sensorik hinsichtlich ihrer Kompatibilität gefordert. Sensoren werden nicht nur beim Bau von Neuanlagen, sondern zunehmend auch zur Optimierung bestehender Anlagen eingesetzt. Die Innovationszyklen für Anlagen und Prozessleittechnik können dabei höchst unterschiedlich sein.

Die neuen Anforderungen an die Prozess-Sensorik gehen über die Erfassung von Prozessinformationen hinaus. Auch Zwischen- und Trendinformationen zu Produkteigenschaften müssen zu Regelzwecken erfasst werden. Weiterhin besteht ein hoher Bedarf nach Informationen 
Zeit für Lösungswege zu einer Anwendungsreife

\begin{tabular}{|c|c|}
\hline$*$ & $*$ \\
bis 2013 & $*$ \\
bis 2020 & nach 2020 \\
\end{tabular}

Anwendungshäufigkeit

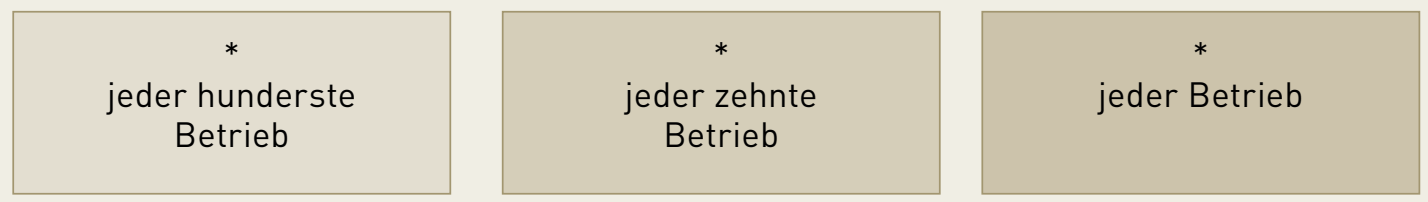

Innovationsgeld

\begin{tabular}{|c|c|c|}
\hline $\begin{array}{c}* \\
\text { verf. Anpassung, } \\
\text { Weiterentwicklung }\end{array}$ & Produktentwicklung & $\begin{array}{c}* \\
\text { Forschungsbedarf }\end{array}$ \\
\hline
\end{tabular}

BILD 1: Bewertung von Lösungsansätzen. Die am häufigsten genannten

Technologien werden bewertet. Es kann somit ein Eindruck über die markt-

bezogene Bedeutung eines Verfahrens gewonnen werden.

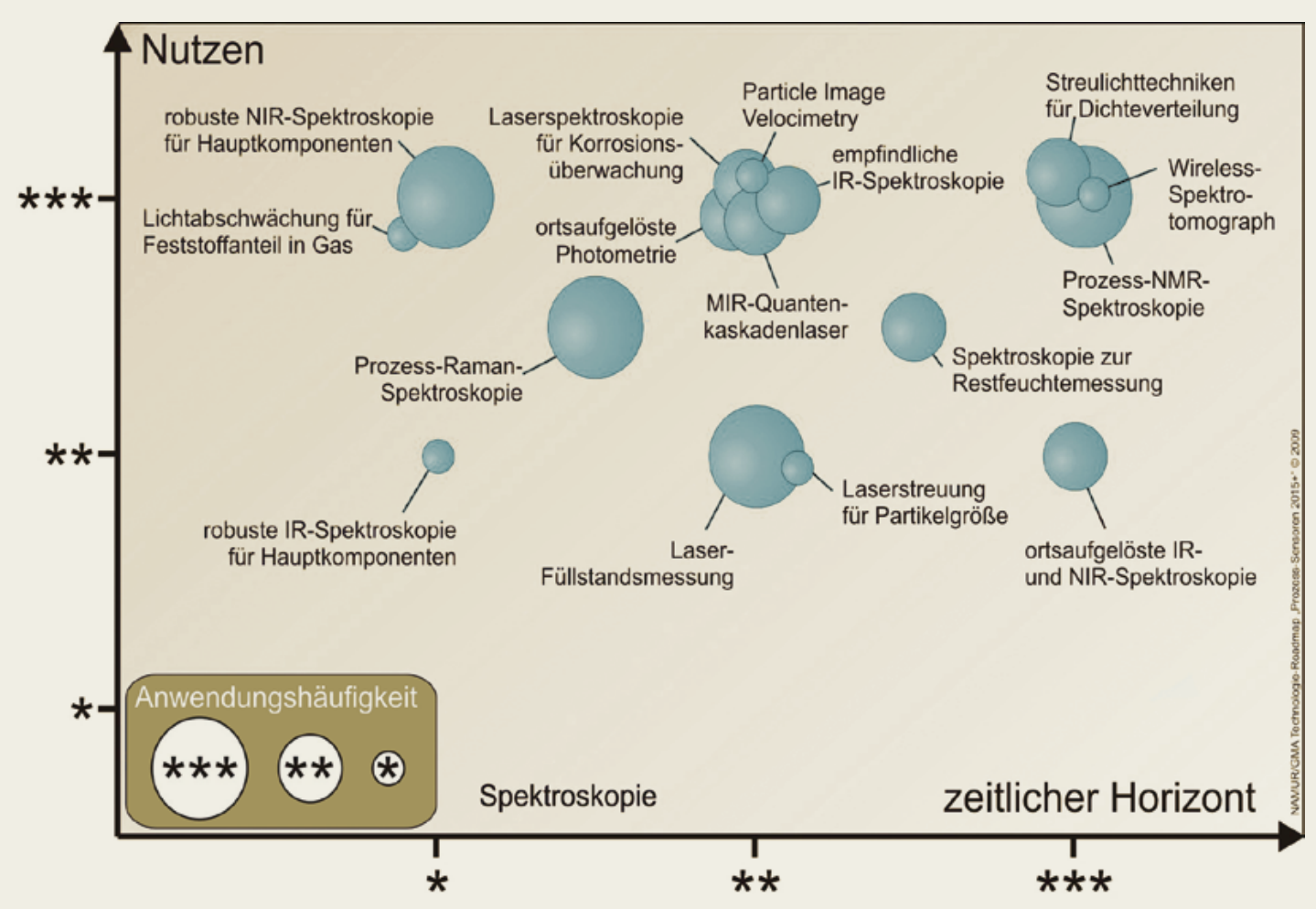

BILD 2: Lösungspotenzial wichtiger Technologien am Beispiel Spektroskopie. Neben der Einordnung mittels der Achsen „Nutzen“ und „zeitlicher Horizont" unterscheidet die Symbolgröße die erwartete Anwendungshäufigkeit. Verfahren mit dem größten Nutzen und dem kürzesten zeitlichen Entwicklungshorizont finden sich im Diagramm links oben. 
über die räumliche Verteilung der verschiedensten Prozessgrößen und zur Ermittlung und Lokalisierung von Grenzflächen und Grenzphasen.

Besonders deutlich wurde bei der Überarbeitung, dass der Trend zu Bioprozessen auch für bisher konventionell chemisch hergestellte Produkte Sensoren verlangt, die heute noch nicht angeboten werden. Das markanteste Beispiel hierfür ist der Bedarf nach einer prozesstauglichen Zielproteinanalyse. Ein neuer Ansatz zeigt auch den Bedarf nach Prozessanalytik mit Einweg-Sensoren im bioverfahrenstechnischen Umfeld auf: Zur Reduktion der Reinigungszeiten von Bioprozessananlagen wird ein Trend zu „disposablen Fermentern“ beobachtet. Ebenso wie die Fermenter müssen dann auch die integrierten Prozess-Sensoren als Einwegsysteme ausgelegt werden. Dieses erfordet entweder eine drastische Verbilligung der Prozess-Sensoren, die Austauschbarkeit der medienberührenden Sensorteile oder ganz neue, nichtinvasive Sensortechnologien.

Eine weitere These zeigt neue Anforderungen an die Prozessmesstechnik durch die zunehmende Verwendung nachwachsender oder recycelter Einsatzstoffe auf. Nachwachsende und recycelte Einsatzstoffe unterliegen hinsichtlich ihrer für die weitere Verarbeitung relevanten Eigenschaften einer wesentlich größeren Bandbreite als bisher. Dies zeigen beispielsweise aktuelle Problemstellungen der Energiewirtschaft bei der Nutzung von Sekundärbrennstoffen oder Biokraftstoffen, woraus sich in erheblichem Maße neue Anforderungen an die Analyse der Einsatzstoffe in diskontinuierlichen Prozessen (beispielsweise Wareneingangskontrolle) und kontinuierlichen Prozessen (z.B. Stoffströme in Rohrleitungen und auf Förderbändern) ableiten lassen.

Der wachsende Bedarf zur Überwachung von Komponenten in Gasen mit immer niedrigeren Erfassungsgrenzen wird heute immer deutlicher. Anwendungsbeispiele sind etwa Komponenten in der Raum- oder Betriebsluft oder Spuren von Sauerstoff in Inert-Prozessen sowie technische Gase als Einsatzstoffe (z.B. Biogase, Gase für Brennstoffzellen), die Katalysatorgifte in kleinen Konzentrationen enthalten können. Daraus resultieren Forderungen nach Messbereichen bis in den niedrigen ppm- oder ppbBereich für einzelne Komponenten. Die heute marktgängigen Technologien wie z.B. elektrochemische Sensoren stoßen diesbezüglich vermutlich an ihre Grenzen.

Die zweifelsfreie Ermittlung der Eigenschaften gelagerter und transportierter Stoffe in logistischen Prozessen ist immer entscheidender für die meisten Produktionsprozesse. Es muss beispielsweise sichergestellt sein, dass nur freigegebene Ware in den Produktionsprozess gelangt und diese sich seit der Beprobung nicht verändert hat. Die Anzahl der Stichproben ist unter anderm aus wirtschaftlichen Gründen begrenzt. Weitere Einschränkungen können sich aus Gründen der Arbeits- und Anlagensicherheit oder der Produktqualität ergeben. Eine nichtinvasive und schnelle Analytik würde diese Nachteile vermeiden. Es gibt einen wachsenden Bedarf an nichtinvasiver Sensorik für die Warenlogistik. Hierzu besteht zunächst ein Bedarf nach neuen analytischen Methoden (z.B. spektroskopische Fingerprintmethoden). Geeignete Messfenster an Transportbehältern und -gebinden oder die Integration von Sensorik in Systeme zur Chargenverfolgung (z.B. RFID) könnten hier eine sichere und handhabbare Warenkontrolle vereinfachen.
Insgesamt lässt sich der wachsende Trend zu InlineMessungen bestätigen. Wenn in einem Prozess Informationen über Stoffeigenschaften mit einer geringen Zeitverzögerung benötigt werden, stellt die Inline-Messung die optimale Form dar. Online-Messungen, die im Bypass erfolgen, sowie Atline-Messungen oder Labormessungen mit Probenentnahme stellen in solchen Fällen immer nur einen Kompromiss dar. Inline-Systeme müssen unter den technischen Bedingungen des Prozesses einsetzbar sein, ohne diesen zu beeinflussen. Zugleich müssen sie die Robustheit konventioneller Sensoren aufweisen. Eine vielfach noch zu lösende Herausforderung der Inline-Messtechnik ist die Kalibrierung und Justierung ohne Prozessbeeinträchtigung.

\subsection{Technologiewünsche}

Aus technologischer Sicht bleiben noch immer große Wünsche offen, für die neuartige Ansätze erforderlich sind. Im folgenden sind drei wesentliche Aussagen herauskristallisiert, die fallweise völlig neue Wirkmechanismen und Technologien bedingen.

n Bestimmung räumlich verteilter Prozessinformationen

- Online-Erfassung völlig neuer Messgrößen (z. B. Konzentration von Sporen in Fermentern)

- Online-Spurenanalytik (Stichwort: Online-ppb)

Die Roadmap zeigt einige aktuelle Verfahren auf, wie Quantenkaskadenlaser für laserbasierte Analysatoren, Rechnertechnik und Miniaturisierung, die das Überführen von Laborspektrometern in Prozess-Spektrometer erlauben, Mikrosystemtechnik mit Realisierungsansätzen für schnellere Chromatographiesäulen (Nanobore-Säulen) oder Mikroverfahrenstechnik mit Ansätzen zur Verbesserung der Probenvorbereitung.

Wenn hier eines Tages technologische Lösungen greifbar sind, werden diese allerdings gleichzeitig zu neuen Paradigmen in der Systemintegration und der Informationsverarbeitung führen. Am Beispiel der Bestimmung räumlich verteilter Prozessgrößen werden sich Fragen nach der Art der Information aus einer räumlicher verteilten Datenmenge, der Art der Visualisierung für den Anlagenfahrer (z.B. als Abweichung von einem definierten Gutzustand) ergeben. Hier geht der Forschungs- und Entwicklungsbedarf deutlich über die Sensortechnologie selbst hinaus.

Die Roadmap zeigt auch auf, dass der Realisierungsbedarf für neuartige Prozess-Sensoren zum großen Teil nicht aufgrund neuer Anforderungen entsteht, sondern weil technologische Weiterentwicklungen die Lösung dieser bereits länger bestehenden Anforderungen heute oder in naher Zukunft ermöglichen.

\subsection{Den größten Nutzen schnell erreichen}

Die genannten technologischen Realisierungsansätze werden ergänzt durch Abschätzungen des noch notwendigen Zeitbedarfs bis zur Anwendungsreife, der Anwendungshäufigkeit und des Nutzens. Sie werden, wie in Bild 1 dargestellt, zwischen einem und drei Punkten bewertet. 
Ferner wird klassifiziert, wie anspruchsvoll die Umsetzung von Lösungen für diese Messaufgabe jeweils sein wird: Handelt es sich um eine neue Anordnung vorhandener Komponenten, die durch eine verfahrenstechnische Anpassung oder gerätetechnische Weiterentwicklung umgesetzt werden kann, oder ist der Neuigkeitsgrad so groß, dass eine Vorentwicklung oder sogar grundlegend ansetzende Forschung notwendig ist?

Die Roadmap greift die verschiedenen Technologieansätze detailliert auf und stellt die Bewertungen graphisch heraus. Bild 2 gibt dieses beispielhaft für spektroskopische Techniken wieder. Um die Aufmerksamkeit und Kraft auf die wichtigsten Entwicklungsfelder zu lenken, lässt sich die Graphik von links oben nach rechts unten lesen, wenn Verfahren mit dem größten Nutzen und dem geringstan zeitlichen Horizont angegangen werden sollen. Die Graphiken werden im Anhang der Roadmap tabellarisch aufgegriffen und mit den Anforderungsbögen verknüpft, um die Detailtiefe auch hier nicht zu verlieren.

Neue Prozess-Sensoren sind darauf angewiesen, auf bestehende oder neue Technologien aus anderen Anwendungen und Industrien zurückzugreifen, wo hohe Stückzahlen die Entwicklung dieser Technologien gerechtfertigt haben. Vorangetrieben aus den Branchen Automobil, Telekommunikation, Multimedia, sowie Medizintechnik entstanden und entstehen eine Fülle neuer Produkte und Technologien, die auch Lösungen für die in dieser Roadmap beschriebenen Anforderungen für die Prozess-Sensorik bieten können. Ein Einsatz modularer Technologie und die Schaffung geeigneter Standards können ebenfalls dazu beitragen, neue ProzessSensorik wirtschaflich und damit attraktiv für Herstel-

\section{AUTOR}

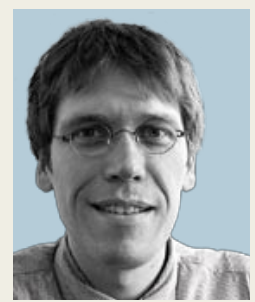

\section{MICHAEL MAIWALD}

(geb. 1967) hat Physikalische Chemie an der Ruhr-Universität Bochum studiert und dort bei Gerhard M. Schneider 1997 promoviert. 1998-2004 war er für den Aufbau und die Leitung der Arbeitsgruppe Online-NMR-Spektroskopie am Institut für Technische Thermodynamik und Thermische Verfahrenstechnik der Universität Stuttgart verantwortlich. 2005-2008 widmete er sich bei der Merck KGaA in Darmstadt der Prozessanalytik im Pharma- und Chemieumfeld. Seit 2008 leitet er eine Fachgruppe „Prozessanalytik“ der BAM in Berlin.

\section{BAM}

Bundesanstalt für Materialforschung und -prüfung, Richard-Willstätter-Str. 11, 12489 Berlin, Tel. +4930 8104-1140,

E-Mail: michael.maiwaldabam.de ler und Markt zu machen. Diese Aufgaben werden am schnellsten erfüllt, wenn Anwender, Hersteller und Forschung gemeinsam voranschreiten.

\section{FAZIT}

Die Technologie-Roadmap zeigt den hohen Bedarf an eine konsequente Weiterentwicklung und ständige Verbesserung der Prozess-Sensorik mit dem Ziel anforderungsgerechter Prozess-Sensoren aus Anwendersicht sehr genau auf. Durch Umsetzung dieser Entwicklungsziele von den Geräteherstellern und der Forschung zusammen mit den Anwendern kann ein erheblicher Wettbewerbsvorteil erzielt werden, der allen zugutekommt. Die Roadmap liefert darüber hinaus Perspektiven für Forschungs- und Entwicklungsförderung und gibt Ansätze für die Normungsarbeit. Sie sollte damit auch für Politik, Industrieverbände und Gremien interessant sein.

Es wird auch das große Innovationspotenzial der Prozessanalysentechnik deutlich, die heute im wesentlichen zur Registrierung und Überwachung und noch nicht konsequent zur sensorgestützen Prozessführung und Prozessoptimierung eingesetzt wird, um damit überlegene Herstellverfahren durch optimierte Produktqualität bei reduzierten Herstellkosten zu ermöglichen.

Neben der technologischen Weiterentwicklung der Prozess-Sensoren darf auch die Erhöhung des Vertrauens in die komplexe Technologie nicht vernachlässigt werden.

MANUSKRIPTEINGANG 02.12.2009

\section{REFERENZEN}

[1] Abele, T.; Kaiser, U.; Drathen, H.; Westerkamp, D. Laube, T.: Automatisierungstechnische Praxis 47 (2005) Heft 8, 36-41.

[2] Abele, T.; Kaiser, U.; Drathen, H. Westerkamp, D.; Fay, U.: Automatisierungstechnische Praxis 47 (2005) Heft 9, 50-54

[3] Babel, W.; Gerlach, M.; Steckenreiter, T.: Fortschritte in der Sensortechnik entlang der Technologie-Roadmap, Automatisierungstechnische Praxis Online (2009) Ausgabe 1-2, 100-107, http://www.atp-online.de

[4] Kloska, M.: Prozessanalysentechnik - Quo Vadis?, 70. NAMUR-Hauptsitzung, Lahnstein, 08. und 09. Nov. 2007.

[5] http://www.namur.de bzw. http://www.vdi.de/gma/prozess-sensoren2015.

\section{DANKSAGUNG}

